Bull. Korean Math. Soc. 49 (2012), No. 3, pp. 635-645

http://dx.doi.org/10.4134/BKMS.2012.49.3.635

\title{
A STUDY ON A RULED SURFACE WITH LIGHTLIKE RULING FOR A NULL CURVE WITH CARTAN FRAME
}

\author{
Nihat Ayyildiz and Tunahan Turhan
}

\begin{abstract}
In this study, we investigate the curvature functions of ruled surface with lightlike ruling for a null curve with Cartan frame in Minkowski 3-space. Also, we give relations between the curvature functions of this ruled surface and curvature functions of central normal surface. Finally, we use the curvature theory of the ruled surface for determine differential properties of a robot end-effector motion.
\end{abstract}

\section{Introduction}

In recent years a number of papers on ruled surfaces and their invariants in Minkowski 3-space have appeared ([2], [9], [11]). Several of these have concern mainly with null scroll and $B$-scroll ([1], [3], [4], [5], [8], [10]). However, there is a gap relative to the curvature theory of ruled surface, whose base curve is null, with lightlike ruling. Here we should note that the ruled surface is neither null scroll nor $B$-scroll. So, aim of this paper is to contribute to this theory.

In Section 3, we give the curvature functions characterized the ruled surface with lightlike ruling for a Cartan framed null curve and also relations between the curvature functions of this ruled surface and curvature functions of central normal surface.

Furthermore, as a rigid body moves in space lines embedded in the body trace ruled surfaces. These lines may be the axes of the joints a spatial mechanisms or manipulators or the line of action of the end-of-arm tooling of a manipulator. The curvature theory of line trajectories seeks to characterize the shape of body carrying the line that generates it ([13], [12]). So, in Section 4 , we shall give end-effector motion in Minkowski 3-space.

Received March 11, 2011.

2010 Mathematics Subject Classification. 53A04, 53A25, 53A40.

Key words and phrases. rigid body, null scroll, Cartan frame, Frenet formulae, striction curve.

This work was supported by Süleyman Demirel University through Scientific Research Project Program (2037-YL-09). 


\section{Preliminaries}

Let $E_{1}^{3}$ be Minkowski 3-space with the inner product

$$
\langle u, v\rangle=-u_{1} v_{1}+u_{2} v_{2}+u_{3} v_{3}
$$

and the vector product

$$
u \times v=\left(-\left|\begin{array}{ll}
u_{2} & u_{3} \\
v_{2} & v_{3}
\end{array}\right|,\left|\begin{array}{ll}
u_{3} & u_{1} \\
v_{3} & v_{1}
\end{array}\right|,\left|\begin{array}{ll}
u_{1} & u_{2} \\
v_{1} & v_{2}
\end{array}\right|\right),
$$

where $u=\left(u_{1}, u_{2}, u_{3}\right), v=\left(v_{1}, v_{2}, v_{3}\right) \in E_{1}^{3}$. For any $u, v, w$ and $z \in E_{1}^{3}$, the Lagrange formula is given by, $([11])$,

$$
\langle u \times v, w \times z\rangle=-\langle u, w\rangle\langle v, z\rangle+\langle u, z\rangle\langle v, w\rangle .
$$

Definition 1. A vector $u$ in $E_{1}^{3}$ is said to be spacelike if $\langle u, u\rangle>0$ or $u=0$, timelike if $\langle u, u\rangle<0$, lightlike or null if $\langle u, u\rangle=0$ and $u \neq 0$.

Definition 2. A parametrized curve $\alpha=\alpha(s)$ in Minkowski 3-space $E_{1}^{3}$ is said to be a null curve if its tangent vector field is null, that is,

$$
\left\langle\alpha^{\prime}(s), \alpha^{\prime}(s)\right\rangle=0, \quad \alpha^{\prime}(s) \neq 0 .
$$

Definition $3([7])$. A base $\mathcal{F}=\{T, N, W\}$ of $E_{1}^{3}$ is called a (proper) null frame if it satisfies the following conditions:

$$
\begin{gathered}
\langle T, T\rangle=\langle N, N\rangle=0,\langle T, N\rangle=1, \\
\langle T, W\rangle=\langle N, W\rangle=0,\langle W, W\rangle=1,
\end{gathered}
$$

where $W=N \times T$.

Let $\alpha=\alpha(s)$ be a null curve in $E_{1}^{3}$. Assume that $T$ is a null vector field along $\alpha$. So, there exists a null vector field $N=N(s)$ along $\alpha$ satisfying $\langle T, N\rangle=1$. Here if we put $W=N \times T$, then we can obtain a (proper) null frame field $\mathcal{F}=\{T, N, W\}$ along $\alpha$. In such a case, the pair $(\alpha, \mathcal{F})$ is said to be a null curve with Cartan frame if it satisfies the following, ([7]),

$$
\begin{aligned}
T^{\prime} & =W, \\
N^{\prime} & =k W, \\
W^{\prime} & =-k T-N,
\end{aligned}
$$

where $k=k(s)$ is smooth function defined by

$$
k=\left\langle N^{\prime}, W\right\rangle .
$$

\section{A ruled surface with lightlike ruling}

Let $(\alpha, \mathcal{F})$ be a null curve with Cartan frame $\mathcal{F}=\{T, N, W\}$. A ruled surface $M$ may be represented by the equation

$$
X(\psi, \nu)=\alpha(\psi)+\nu V(\psi),
$$

where $\psi$ and $v$ are arbitrary real valued parameters. The curve $\alpha(\psi)$ is called the directrix of $M$ and $V(\psi)$ is a vector attached to the straight line generating 
the ruled surface. The Darboux frame at $\alpha(\psi)$ is the basis $\{T, V, U\}$ of $E_{1}^{3}$, where $T$ is the tangent vector of the curve $\alpha, V$ is the unique vector obtained by, $([7])$,

$$
V=\frac{1}{\langle X, T\rangle}\left\{X-\frac{\langle X, X\rangle}{2\langle X, T\rangle} T\right\}, \quad X \in T_{\alpha(\psi)} M, \quad\langle X, T\rangle \neq 0,
$$

and $U$ is the spacelike unit normal of $M$ which is defined by $U=V \times T$. Hence, we have

$$
\langle T, T\rangle=\langle V, V\rangle=\langle V, U\rangle=\langle T, U\rangle=0,\langle T, V\rangle=1,\langle U, U\rangle=1 .
$$

Thus we have the following result:

Theorem 3.1 ([6]). Let $\alpha$ be a null curve in $M \subset E_{1}^{3}$. The first order variation of Darboux frame $\{T, V, U\}$ is

$$
\left[\begin{array}{l}
T^{\prime} \\
V^{\prime} \\
U^{\prime}
\end{array}\right]=\left[\begin{array}{ccc}
\kappa_{g} & 0 & \kappa_{n} \\
0 & -\kappa_{g} & \tau_{g} \\
-\tau_{g} & -\kappa_{n} & 0
\end{array}\right]\left[\begin{array}{l}
T \\
V \\
U
\end{array}\right]
$$

where $\left\langle T^{\prime}, V\right\rangle=\kappa_{g},\left\langle T^{\prime}, U\right\rangle=\kappa_{n}$ and $\left\langle V^{\prime}, U\right\rangle=\tau_{g}$.

These functions $\kappa_{g}, \kappa_{n}$ and $\tau_{g}$ are called the geodesic curvature, the normal curvature and the geodesic torsion of the curve $\alpha$, respectively.

So, a relation between a null curve with Cartan frame $\{T, N, W\}$ and Darboux frame $\{T, V, U\}$ of a ruled surface $M$ is given by:

Theorem 3.2. Let $M$ be a ruled surface with basis $\{T, V, U\}$ in $E_{1}^{3}$ and $\alpha$ be a null curve with Cartan frame $\{T, N, W\}$ in $M \subset E_{1}^{3}$. Then

$$
\left[\begin{array}{l}
T \\
N \\
W
\end{array}\right]=\left[\begin{array}{ccc}
1 & 0 & 0 \\
-\frac{\kappa_{g}^{2}}{\kappa_{n}\left(1+\kappa_{n}\right)} & 1 & -\frac{\kappa_{g}}{\kappa_{n}} \\
\kappa_{g} & 0 & \kappa_{n}
\end{array}\right]\left[\begin{array}{c}
T \\
V \\
U
\end{array}\right]
$$

Proof. If we use equations (1), (2) and the Lagrange formula we get equation (3).

Using this result, it is easy to see that

$$
1=\kappa_{n} .
$$

Substituting equation (4) into equation (3) it gives (see [6])

$$
\left[\begin{array}{l}
T \\
N \\
W
\end{array}\right]=\left[\begin{array}{ccc}
1 & 0 & 0 \\
-\frac{\kappa_{g}^{2}}{2} & 1 & -\kappa_{g} \\
\kappa_{g} & 0 & 1
\end{array}\right]\left[\begin{array}{l}
T \\
V \\
U
\end{array}\right]
$$

Now, we shall give a theorem with respect to the striction line which gives the simplest description of the positional variation of the ruled surface. 
Theorem 3.3. Let $M$ be a ruled surface with basis $\{T, V, U\}$ in $E_{1}^{3}$ and $\alpha$ be a null curve with Cartan frame $\{T, N, W\}$ in $M \subset E_{1}^{3}$. Then the location of the striction line relative to the directrix along the ruling $V$ is

$$
\beta(s)=\alpha(s)-\left(-\frac{1}{2} \int \frac{\kappa_{g}^{2}}{\tau_{g}^{2}} d t+c\right) V(s),
$$

where $c$ is any constant.

Proof. The location of the striction line relative to the directrix along the ruling $V$ is given by

$$
\beta(s)=\alpha(s)-\mu V(s),
$$

where $\mu$ is a real valued parameter. To determine the parameter $\mu$, if we use the definition of the striction line, we get

$$
\mu=-\frac{\kappa_{g}}{\tau_{g}^{2}} .
$$

So, the first order positional variation of the striction line is expressed as

$$
\beta^{\prime}=\Omega T-\Gamma V+\Delta U,
$$

where $\Omega=1, \Gamma=\mu^{\prime}+\frac{\kappa_{g}^{2}}{\tau_{g}^{2}}$ and $\Delta=\frac{\kappa_{g}}{\tau_{g}}$. Here, supposing $\beta$ null vector and taking (7) into consideration, we have

$$
\mu=-\frac{1}{2} \int \frac{\kappa_{g}^{2}}{\tau_{g}^{2}} d t+c
$$

where $c$ is any constant. So, from equation (6) we get

$$
\beta(s)=\alpha(s)-\left(-\frac{1}{2} \int \frac{\kappa_{g}^{2}}{\tau_{g}^{2}} d t+c\right) V(s) .
$$

In this theorem, the functions $\Omega, \Gamma$ and $\Delta$ characterize the ruled surface $M$. These functions are called the curvature functions of the ruled surface $M$.

As the trihedron $T, V, U$ moves along the striction curve of $M$ the two Lorentzian vectors $T$ and $U$ generate ruled surfaces associated with $M$. Of primary importance is the ruled surface generated by $U$ called the central normal surface of $M$. Now let us consider the central normal surface $\widetilde{M}$ which suppose $\beta$ as a base curve and whose spacelike ruling is $U$. We can write its equation

$$
X_{U}(s, \nu)=\beta(s)+\nu U(s) .
$$

Then, the location of the striction line of this surface is given by the following result: 
Theorem 3.4. Let $\widetilde{M}$ be a central normal surface with spacelike ruling $U$ and $\beta$ be its base curve. Then the location of the striction line of the central normal surface is

$$
\beta_{U}(s)=\beta(s)-\frac{\kappa_{g}^{2}-2 \kappa_{n} \tau_{g}}{4 \tau_{g} \kappa_{n}} U(s) .
$$

Proof. From the definition of the striction line we can write

$$
\beta_{U}(s)=\beta(s)-\mu_{U} U(s),
$$

where $\mu_{U}$ is a real parameter. By direct computation the parameter $\mu_{U}$ is

$$
\mu_{U}=\frac{\kappa_{g}^{2}-2 \kappa_{n} \tau_{g}}{4 \tau_{g} \kappa_{n}}
$$

When this is substituted equation (8), the proof is completed.

Using this result, the first-order positional variation of the striction line of the central normal surface $\widetilde{M}$ can be expressed with respect to Cartan frame $\mathcal{F}=\{T, N, W\}$ as follows

$$
\beta_{u}^{\prime}=\Omega_{U} T+\Gamma_{U} N+\Delta_{U} W,
$$

where

$$
\begin{gathered}
\Omega_{U}=\kappa_{g}^{2}\left(\frac{\mu_{U}}{2}-\frac{1}{4} \Delta^{2}\right)-\kappa_{g}\left(\Delta-\mu_{U}^{\prime}\right)+\mu_{U} \tau_{g}+\Omega, \\
\Gamma_{U}=\mu_{U}-\frac{1}{2} \Delta^{2} \text { and } \Delta_{U}=\Delta-\mu_{U}^{\prime}+\kappa_{g}\left(\mu_{U}-\frac{1}{2} \Delta^{2}\right) .
\end{gathered}
$$

The functions $\Omega_{U}, \Gamma_{U}$ and $\Delta_{U}$ characterize the central normal surface $\widetilde{M}$. These functions are called the curvature functions of the central normal surface $\widetilde{M}$.

Now, we would like to exhibit an example for these surfaces:

Example 1. Let the ruled surface $M$ be given by

$$
X(s, t)=(-s, \cos s, \sin s)+t(1, \cos s, \sin s),
$$

where the null curve $\alpha(s)=(-s, \cos s, \sin s)$ is the directrix of the surface and $V=(1, \cos s, \sin s)$ is the ruling vector of the surface $M$ (see Figure 1). So, we can construct the Darboux frame of the surface $M$ as follows:

$$
\begin{aligned}
& T=(-1,-\sin s, \cos s), V=(1, \cos s, \sin s) \text { and } \\
& U=(-1,-\sin s-\cos s,-\sin s+\cos s) .
\end{aligned}
$$

The vectors $T, V$ and $U$ satisfy the following conditions:

$$
\langle T, T\rangle=\langle V, V\rangle=0,\langle T, V\rangle=\langle U, U\rangle=1,\langle V, U\rangle=\langle T, U\rangle=0, U=V \times T .
$$

Hence, we get the Cartan frame $\mathcal{F}=\{T, N, W\}$ of the null curve $\alpha$ as $T=(-1,-\sin s, \cos s), N=\left(\frac{1}{2},-\frac{\sin s}{2}, \frac{\cos s}{2}\right)$ and $W=(0,-\cos s,-\sin s)$. 


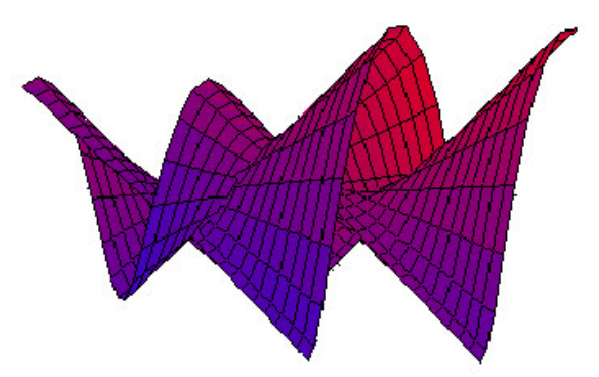

Figure 1. The ruled surface $X(s, t)$ with lightlike ruling $V$.

Therefore, a Cartan framed null curve $(\alpha, \mathcal{F})$ satisfies the following Frenet equations

$$
\begin{aligned}
T^{\prime} & =W, \\
N^{\prime} & =\frac{1}{2} W, \\
W^{\prime} & =-\frac{1}{2} T-N .
\end{aligned}
$$

On the other hand, the first order variation of the Darboux frame $\{T, V, U\}$ can be expressed as

$$
\begin{aligned}
& T^{\prime}=-T+U, \\
& V^{\prime}=V+U, \\
& U^{\prime}=-T-V .
\end{aligned}
$$

So, the curvature functions of the ruled surface $M$ can be obtained as, respectively,

$$
\Delta=-1, \Gamma=1 \text { and } \Omega=1 .
$$

Now, let us assume that the equation of the central normal surface $\widetilde{M}$ with spacelike ruling (see Figure 2) is given by

$$
X_{U}(s, t)=(-s, \cos s, \sin s)+t(-1,-\sin s-\cos s,-\sin s+\cos s) .
$$

Therefore, the geodesic curvature $\kappa_{g}$, the normal curvature $\kappa_{n}$ and the geodesic torsion $\tau_{g}$ of the curve $\alpha=(-s, \cos s, \sin s)$ are found as

$$
\kappa_{g}=-1, \kappa_{n}=1 \text { and } \tau_{g}=1 .
$$

Finally, the curvature functions of the central normal surface $\widetilde{M}$ can be obtained as follows, respectively,

$$
\Delta_{U}=-\frac{1}{4}, \Gamma_{U}=-\frac{3}{4} \text { and } \Omega_{U}=-\frac{5}{8} .
$$




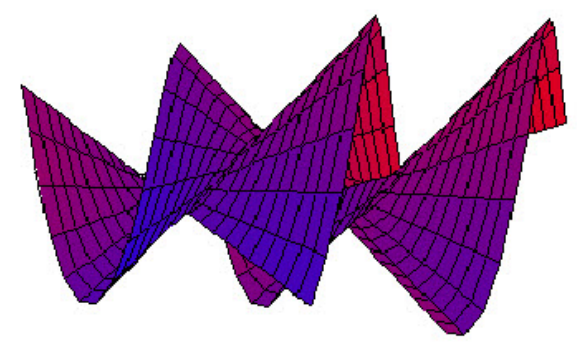

FiguRE 2. The central normal surface $X_{U}(s, t)$ with spacelike ruling $U$.

\section{END-effector motion}

A typical robot trajectory is shown in Figure 3. Also, there are three vector $\{\overline{0}, \bar{A}, \bar{N}\}$. The tool frame consists of these three vector. Each of the these three vector of the tool frame generates a ruled surface. It is not necessary to use all three ruled surfaces to represent a robot trajectory ([14]). One ruled surface will suffice. The ruled surface generated by the $\overline{0}$ is chosen here to represent the trajectory. There are four frame of reference which are essential

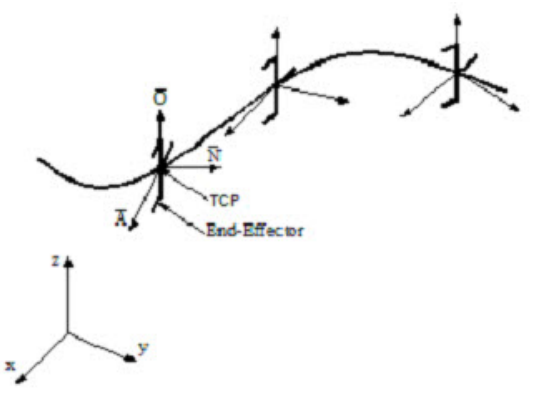

Figure 3. A robot trajectory.

in the study of the motion of the end-effector. The four frames are: the tool frame $\{\overline{0}, \bar{A}, \bar{N}\}$, the surface frame $\left\{\overline{0}, S_{n}, S_{b}\right\}$, Darboux frame $\{T, V, U\}$ and Cartan frame $\{T, N, W\}$ ([14]). The surface frame $\left\{\overline{0}, S_{n}, S_{b}\right\}$ and the tool frame $\{\overline{0}, \bar{A}, \bar{N}\}$ satisfy the following, respectively,

$$
\begin{aligned}
\overline{0} & =V, \\
S_{b} & =\frac{1}{\langle Y, V\rangle}\left\{Y-\frac{\langle Y, Y\rangle}{2\langle Y, V\rangle} V\right\}, \quad Y \in T_{\alpha(t)} M, \quad\langle Y, V\rangle \neq 0,
\end{aligned}
$$




$$
\begin{aligned}
S_{n} & =S_{b} \times \overline{0}, \\
\langle\overline{0}, \overline{0}\rangle & =\left\langle S_{b}, S_{b}\right\rangle=\left\langle\overline{0}, S_{n}\right\rangle=\left\langle S_{b}, S_{n}\right\rangle=0,\left\langle\overline{0}, S_{b}\right\rangle=\left\langle S_{n}, S_{n}\right\rangle=1
\end{aligned}
$$

and

$$
\begin{aligned}
\langle\overline{0}, \overline{0}\rangle & =\langle\bar{A}, \bar{A}\rangle=\langle\bar{A}, \bar{N}\rangle=\langle\overline{0}, \bar{N}\rangle=0, \\
\langle\overline{0}, \bar{A}\rangle & =\langle\bar{N}, \bar{N}\rangle=1, \\
\bar{N} & =\bar{A} \times \overline{0} .
\end{aligned}
$$

Now we can express relation between the tool frame $\{\overline{0}, \bar{A}, \bar{N}\}$ and the surface frame $\left\{\overline{0}, S_{n}, S_{b}\right\}$ in matrix form as

$$
\left[\begin{array}{c}
\overline{0} \\
\bar{A} \\
\bar{N}
\end{array}\right]=\left[\begin{array}{ccc}
1 & 0 & 0 \\
\left\langle\bar{A}, S_{b}\right\rangle & \left\langle\bar{A}, S_{n}\right\rangle & 1 \\
\left\langle\bar{N}, S_{b}\right\rangle & \left\langle\bar{N}, S_{n}\right\rangle & 0
\end{array}\right]\left[\begin{array}{c}
\overline{0} \\
S_{n} \\
S_{b}
\end{array}\right] .
$$

The relation between the surface frame $\left\{\overline{0}, S_{n}, S_{b}\right\}$ and Darboux frame $\{T, V, U\}$ is expressed in matrix form as

$$
\left[\begin{array}{c}
\overline{0} \\
S_{n} \\
S_{b}
\end{array}\right]=\left[\begin{array}{ccc}
0 & 1 & 0 \\
0 & \left\langle S_{n}, T\right\rangle & -1 \\
1 & \left\langle S_{b}, V\right\rangle & \left\langle S_{b}, U\right\rangle
\end{array}\right]\left[\begin{array}{c}
T \\
V \\
U
\end{array}\right] .
$$

Lastly, if we use equation (11) into equation (10), we can express relation between the tool frame $\{\overline{0}, \bar{A}, \bar{N}\}$ and Darboux frame $\{T, V, U\}$ as

$$
\left[\begin{array}{c}
\overline{0} \\
\bar{A} \\
\bar{N}
\end{array}\right]=\left[\begin{array}{lll}
0 & 1 & 0 \\
1 & l_{1} & l_{2} \\
0 & l_{3} & l_{4}
\end{array}\right]\left[\begin{array}{l}
T \\
V \\
U
\end{array}\right],
$$

where

$$
\begin{aligned}
& l_{1}=\left\langle S_{b}, \bar{A}+T\right\rangle+\left\langle\bar{A}, S_{n}\right\rangle\left\langle S_{n}, T\right\rangle, \\
& l_{2}=\left\langle S_{b}, U\right\rangle-\left\langle\bar{A}, S_{n}\right\rangle, \\
& l_{3}=\left\langle\bar{N}, S_{b}\right\rangle+\left\langle\bar{N}, S_{n}\right\rangle\left\langle S_{n}, T\right\rangle, \\
& l_{4}=-\left\langle\bar{N}, S_{n}\right\rangle .
\end{aligned}
$$

Now we may express differential properties of the robot end-effector motion in the Darboux frame and tool frame, respectively,

$$
\alpha^{\prime}=T-\left(\Gamma-\mu^{\prime}+\kappa_{g} \mu\right) V+\left(\Delta+\mu \tau_{g}\right) U
$$

and

$$
\alpha^{\prime}=\Lambda_{1} \overline{0}+\bar{A}+\Lambda_{2} \bar{N}
$$

where

$$
\Lambda_{1}=\frac{\mu^{\prime} l_{4}-l_{4} \Gamma-l_{4} l_{1}+l_{3} l_{2}-\mu \kappa_{g} l_{4}-l_{3} \Delta-\mu \tau_{g} l_{3}}{l_{4}} \text { and } \Lambda_{2}=\frac{\mu \tau_{g}-l_{2}+\Delta}{l_{4}} .
$$


To determine the first-order variation of the tool frame, we differentiate equation (12) to get

$$
\frac{d}{d s}\left[\begin{array}{c}
\overline{0} \\
\bar{A} \\
\bar{N}
\end{array}\right]=\left[\begin{array}{ccc}
0 & -\kappa_{g} & \tau_{g} \\
-l_{2} \tau_{g} & l_{1}^{\prime}-l_{1} \kappa_{g}-l_{2} \kappa_{n} & l_{2}^{\prime}+l_{1} \tau_{g} \\
-l_{4} \tau_{g} & l_{3}^{\prime}-l_{3} \tau_{g}-l_{4} \kappa_{n} & l_{4}^{\prime}+l_{3} \tau_{g}
\end{array}\right]\left[\begin{array}{c}
T \\
V \\
U
\end{array}\right],
$$

where

$$
\begin{aligned}
l_{1}^{\prime}= & \left\langle S_{b}^{\prime}, \bar{A}+T\right\rangle+\left\langle S_{b}, \bar{A}^{\prime}+T^{\prime}\right\rangle+\left(\left\langle\bar{A}^{\prime}, S_{n}\right\rangle+\left\langle\bar{A}, S_{n}^{\prime}\right\rangle\right)\left\langle S_{n}, T\right\rangle \\
& +\left\langle\bar{A}, S_{n}\right\rangle\left(\left\langle S_{n}^{\prime}, T\right\rangle+\left\langle S_{n}, T\right\rangle\right), \\
l_{2}^{\prime}= & \left\langle S_{b}^{\prime}, U\right\rangle+\left\langle S_{b}, U\right\rangle-\left\langle\bar{A}^{\prime}, S_{n}\right\rangle-\left\langle\bar{A}, S_{n}^{\prime}\right\rangle, \\
l_{3}^{\prime}= & \left\langle\bar{N}^{\prime}, S_{b}\right\rangle+\left(\left\langle\bar{N}^{\prime}, S_{n}\right\rangle+\left\langle\bar{N}, S_{n}^{\prime}\right\rangle\right)\left\langle S_{n}, T\right\rangle+\left\langle\bar{N}, S_{n}\right\rangle\left(\left\langle S_{n}^{\prime}, T\right\rangle+\left\langle S_{n}, T^{\prime}\right\rangle\right), \\
l_{4}^{\prime}= & -\left\langle\bar{N}^{\prime}, S_{n}\right\rangle-\left\langle\bar{N}, S_{n}^{\prime}\right\rangle .
\end{aligned}
$$

An example for the relations defined above may be given as:

Example 2. Let the ruled surface be given by

$$
X(s, t)=(-s, \cos s, \sin s)+t(1, \cos s, \sin s),
$$

where the null curve $\alpha(s)=(-s, \cos s, \sin s)$ is the directrix of the surface and $V=(1, \cos s, \sin s)$ is the ruling vector of the surface $X(s, t)$ (see Figure 1). So, we can construct the surface frame $\left\{\overline{0}, S_{n}, S_{b}\right\}$ as follows:

$$
\begin{aligned}
& \overline{0}=V=(1, \cos s, \sin s), S_{b}=(-5,3 \sin s-4 \cos s,-3 \cos s-4 \sin s) \text { and } \\
& S_{n}=(-3, \sin s-3 \cos s,-3 \sin s-\cos s) .
\end{aligned}
$$

The vectors $\overline{0}, S_{b}$ and $S_{n}$ satisfy the following conditions:

$$
\begin{gathered}
\langle\overline{0}, \overline{0}\rangle=\left\langle S_{b}, S_{b}\right\rangle=0,\left\langle\overline{0}, S_{b}\right\rangle=\left\langle S_{n}, S_{n}\right\rangle=1, \\
\left\langle\overline{0}, S_{n}\right\rangle=\left\langle S_{b}, S_{n}\right\rangle=0, S_{n}=S_{b} \times \overline{0} .
\end{gathered}
$$

Similarly, we can construct the tool frame $\{\overline{0}, \bar{A}, \bar{N}\}$ as follows:

$$
\begin{aligned}
\overline{0} & =V=(1, \cos s, \sin s), \bar{A}=(-13,5 \sin s-12 \cos s,-5 \cos s-12 \sin s) \text { and } \\
\bar{N} & =(-5, \sin s-5 \cos s,-\cos s-5 \sin s) .
\end{aligned}
$$

The vectors $\overline{0}, \bar{A}$ and $\bar{N}$ satisfy the following conditions:

$$
\begin{gathered}
\langle\overline{0}, \overline{0}\rangle=\langle\bar{A}, \bar{A}\rangle=0,\langle\overline{0}, \bar{A}\rangle=\langle\bar{N}, \bar{N}\rangle=1, \\
\langle\overline{0}, \bar{N}\rangle=\langle\bar{A}, \bar{N}\rangle=0, \bar{N}=\bar{A} \times \overline{0} .
\end{gathered}
$$

Hence, from equation (10), we get relation between the tool frame $\{\overline{0}, \bar{A}, \bar{N}\}$ and the surface frame $\left\{\overline{0}, S_{n}, S_{b}\right\}$ in matrix form as

$$
\left[\begin{array}{l}
\overline{0} \\
\bar{A} \\
\bar{N}
\end{array}\right]=\left[\begin{array}{ccc}
1 & 0 & 0 \\
-2 & 2 & 1 \\
-2 & 1 & 0
\end{array}\right]\left[\begin{array}{c}
\overline{0} \\
S_{n} \\
S_{b}
\end{array}\right]
$$


From equation (11), the relation between the surface frame $\left\{\overline{0}, S_{n}, S_{b}\right\}$ and Darboux frame $\{T, V, U\}$ is expressed in matrix form as

$$
\left[\begin{array}{c}
\overline{0} \\
S_{n} \\
S_{b}
\end{array}\right]=\left[\begin{array}{ccc}
0 & 1 & 0 \\
0 & -4 & -1 \\
1 & 1 & -4
\end{array}\right]\left[\begin{array}{l}
T \\
V \\
U
\end{array}\right] .
$$

Lastly, if we use equation (11) into equation (10), we can express relation between the tool frame $\{\overline{0}, \bar{A}, \bar{N}\}$ and Darboux frame $\{T, V, U\}$ in matrix form as

$$
\left[\begin{array}{l}
\overline{0} \\
\bar{A} \\
\bar{N}
\end{array}\right]=\left[\begin{array}{ccc}
0 & 1 & 0 \\
1 & -9 & -6 \\
0 & -6 & -1
\end{array}\right]\left[\begin{array}{l}
T \\
V \\
U
\end{array}\right] .
$$

To determine the first-order variation of the tool frame, we differentiate equation (13) and use equation (9), we get

$$
\frac{d}{d s}\left[\begin{array}{c}
\overline{0} \\
\bar{A} \\
\bar{N}
\end{array}\right]=\left[\begin{array}{ccc}
0 & 1 & 1 \\
5 & -3 & -8 \\
1 & -5 & -6
\end{array}\right]\left[\begin{array}{l}
T \\
V \\
U
\end{array}\right] .
$$

\section{References}

[1] L. J. Alias, A. Ferrandez, P. Lucas, and M. A. Moreno, On the Gauss map of B-scrolls, Tsukuba J. Math. 22 (1998), no. 2, 371-377.

[2] N. Ayyıldız and A. Yücesan, On the scalar and dual formulations of the curvature theory of line trajectories in the Lorentzian space, J. Korean Math. Soc. 43 (2006), no. 6, 1339-1355.

[3] H. Balgetir, M. Bektaş, and M. Ergüt, Null scroll in the 3-dimensional Lorentzian space, Appl. Sci. 5 (2003), no. 1, 1-5.

[4] W. B. Bonnor, Null hypersurfaces in Minkowski space-time, Tensor (N. S.) 24 (1972), $329-345$.

[5] S. M. Choi, U. H. Ki, and Y. J. Suh, On the Gauss map of null scrolls, Tsukuba J. Math. 22 (1998), no. 1, 273-279.

[6] A. C. Çöken and Ü. Çiftçi, On null curves on surfaces and null vectors in Lorentz space, SDU Fen Derg. 2 (2007), no. 1, 111-116.

[7] K. L. Duggal and A. Bejancu, Lightlike Submanifolds of Semi-Riemannian Manifolds and Applications, Kluwer Academic Publishers, 1996.

[8] S. Ersoy and M. Tosun, On the trajectory null scrolls in 3-dimensional Minkowski spacetime $E_{1}^{3}$, Kyungpook Math. J. 48 (2008), no. 1, 81-92.

[9] A. Ferrandez and P. Lucas, On surfaces in the 3-dimensional Lorentz-Minkowski space, Pacific J. Math. 152 (1992), no. 1, 93-100.

[10] A. Ferrandez, A. Gimenez, and P. Lucas, Null helices in Lorentzian space forms, Internat. J. Modern Phys. A. 16 (2001), no. 30, 4845-4863.

[11] H. Liu, Ruled surfaces with lightlike ruling in 3-Minkowski space, J. Geom. Phys. 59 (2009), no. 1, 74-78.

[12] J. M. McCarthy, On the scalar and dual formulations of the curvature theory of line trajectories, Journal of Mechanisms, Transmissions, and Automation in Design 109 (1987), 101-106.

[13] J. M. McCarthy and B. Roth, The curvature theory of line trajectories in spatial kinematics, ASME Journal of Mechanical Design 103 (1981), no. 4, 718-724. 
[14] B. S. Ryuh, Robot trajectory planning using the curvature theory of ruled surfaces, Doctoral dissertation, Purdue University, West Lafayette, Ind, USA, 1989.

Nihat AyYildiz

Department of Mathematics

SÜLEYMAN Demirel University

32260 Isparta, TURKey

E-mail address: nihatayyildiz@sdu.edu.tr, ayyildiz67@gmail.com

Tunahan Turhan

Seydisehir Vocational School

SelÇUK UNiVersity

42360 Konya, TURKEY

E-mail address: t_turhan07@hotmail.com 